\title{
NUEVO REGISTRO DE FLORA PARA LAS LOMAS DE LACHAY (LIMA, PERÚ): PRIMER REPORTE DE Lemna minuta Kunth (ARACEAE)
}

\section{NEW RECORD FOR LOMAS DE LACHAY (LIMA, PERU): FIRST RECORD OF Lemna minuta Kunth (ARACEAE)}

\author{
Héctor Aponte ${ }^{1,2}$
}

\begin{abstract}
Resumen
Se reporta la presencia de una población de Lemna minuta en las Lomas de Lachay. Esta especie no se encuentra actualmente en el listado de plantas de ninguna loma costera y corresponde al primer reporte de una planta acuática flotante en un ecosistema estacional como este. La población de esta especie ha sido observada ocupando uno de los pocos cuerpos de agua en dos años consecutivos. Se discuten las posibles formas de dispersión de la especie hacia este ecosistema de tan marcada estacionalidad.
\end{abstract}

Palabras clave: dispersión, Lemna, lenteja de agua, lomas, planta acuática.

\begin{abstract}
The presence of a population of Lemna minuta in Lomas de Lachay is reported. This species is not currently in the list of plants of any lomas formation and corresponds to the first report of a floating aquatic plant in a seasonal ecosystem like this. The population of this species has been observed occupying one of the few bodies of water in two consecutive years. Possible ways of dispersal of this species to this ecosystem are discussed.
\end{abstract}

Key words: aquatic plants, dispersion, duckweed, Lemna, lomas formation.

\section{Introducción.}

Dentro de las angiospermas acuáticas del Perú se encuentran las plantas del género Lemna L.; género ubicado en la familia Araceae dentro de la subfamilia Lemnoideae (APG III, 2009). Este género tiene cuatro especies reportadas en Perú (L. aequinoctialis Welw.; L. gibba L.; L. minuta Kunth y L. valdiviana Phil.), que ocupan hábitats inundados en las zonas costeras, andinas y amazónicas; dos de ellas, L. gibba L. y $L$. minuta, han sido reportadas para Lima (Brako \& Zarucchi, 1993). Por su rápido crecimiento, algunas especies de este género (como $L$. minuta) tienen un potencial invasivo pudiendo afectar a otras especies acuáticas (Vilà \& Weiner, 2004).

Las lomas costeras de Perú y Chile se caracterizan por ser ecosistemas con una estacionalidad muy marcada. Aquí habitan un aproximado de 847 especies de plantas vasculares adaptadas a condiciones de humedad temporal y extrema aridez (Dillon et al., 2011). Hasta la fecha ninguna especie del género Lemna ha sido reportada para una loma costera. En la costa central del Perú, las lomas se encuentran cerca a los humedales costeros (Figura 1), por lo que se ha propuesto que conforman un corredor de diversidad biológico de la costa Peruana (Aponte et al., 2012). Por su cercanía, es probable que algunas especies acuáticas como Lemna puedan dispersarse entre estos ecosistemas por medio de ornitocoria, fenómeno que no ha sido reportado para las lomas de Lima, pero que si ha sido identificado en otros ecosistemas del mundo (Green et al., 2008).

Una de las lomas más importantes en el Departamento de Lima son las Lomas de Lachay (Provincia de Huaura, Lima, Perú). Este ecosistema pertenece al conjunto de áreas protegida por el estado peruano y alberga 146 especies de plantas vasculares (Cano et al., 1999). En esta localidad existen algunos cuerpos de agua, que podrían albergar a organismos acuáticos, inclusive durante el periodo seco de las lomas.

El presente trabajo reporta la presencia de Lemna minuta en uno de los cuerpos de agua de las Lomas de Lachay.

\section{Materiales y métodos.}

Se realizaron visitas a las Lomas de Lachay en octubre y noviembre del 2014 y noviembre del 2015, durante la estación húmeda. En ellas fue posible apreciar una población de Lemna minuta (ARACEAE, Lemnoideae) ocupando uno de los cuerpos de agua denominado El Puquio (1121'39.88's 77²1'43.18'’W, 385 msnm.). Este punto corresponde a una fuente de agua de no más de 3 metros cuadrados, que está rodeada por rocas que, junto al 


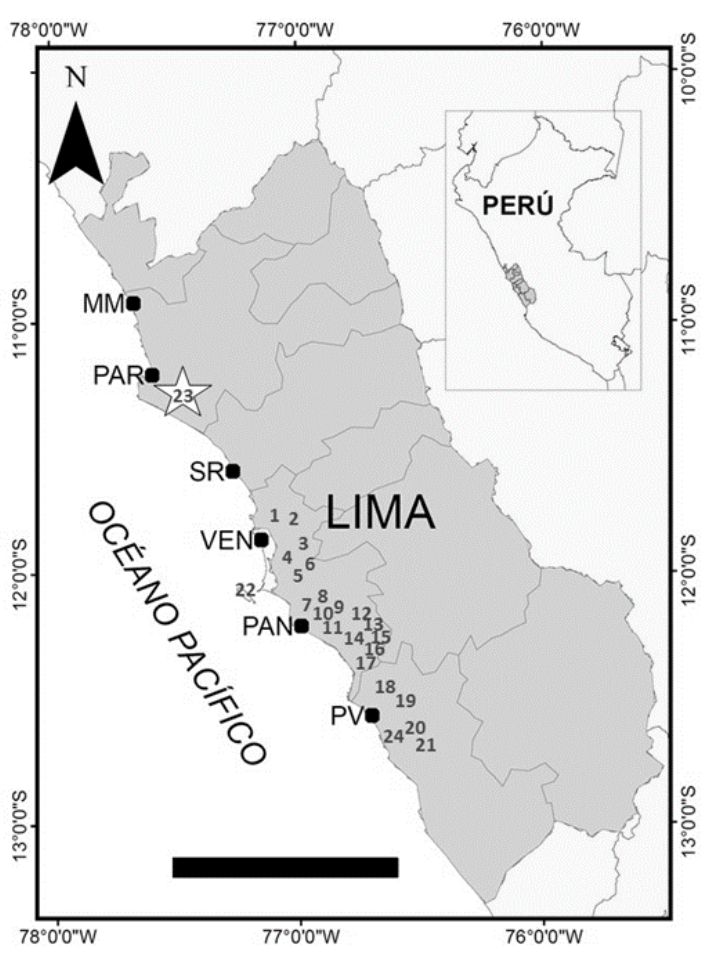

Figura 1. Mapa de Lima y Callao mostrando los principales humedales, lomas y el Tillandsial de Mala. MM=Albuferas de Medio Mundo, PAR=Laguna el Paraíso, SR=Humedal de Santa Rosa, VEN=Humedales de Ventanilla, PAN=Pantanos de Villa, PV=Humedales de Puerto Viejo. Las lomas están numeradas: 1=Carabaylo, 2=Puquio, 3=Collique, 4=Payet, 5=Amancaes, 6=Mangomarca, 7=Villa María, 8=Retamal, 9=Manchay, 10=Zorritos, 11=Lúcumo, 12=Pachacamac, 13=Portillo, 14=Pacta, 15=Malanche, 16=Caringa, 17=Cicasos, 18=Paloma, 19=Lapa Lapa, 20=Mala, 21=Asia, 22=San Lorenzo, 23=Lomas de Lachay. 24= Tillandsial de Piedra Campana. Escala $100 \mathrm{~km}$.

suelo limo - arenoso, permiten la acumulación de agua. La muestra fue colectada y herborizada con técnicas estándar (Bridson \& Forman, 1998) y se utilizaron claves taxonómicas y descripciones a fin de corroborar la identificación de la especie (León 1993, Armstrong 2013). Finalmente la planta fue depositada en el herbario USM.

\section{Resultados.}

Lemna minuta Kunth

En el área de estudio, la población de esta especie está compuesta por varias centenas de frondas que ocupan de manera parcial el cuerpo de agua y la zona húmeda de los alrededores (Figura 2). En las ocasiones en que se observó una menor cantidad de agua en El Puquio, las plantas de L. minuta ocupaban el suelo húmedo, mostrándose en buen estado a pesar de dicha disminución. La frondas tenían parénquima aerífero de poco espesor (ausencia de giba) y se encontraban formando grupos de dos o tres. La nervadura tuvo un largo promedio de $0.62 \mathrm{~mm}$ (mínimo=0.37; máximo=0.85) sin sobrepasar los dos tercios de la distancia entre el nudo y el ápice de la fronda. Cada fronda presentó una raíz. El largo de la fronda promedio fue de $1.62 \mathrm{~mm}$ (mínimo=1.05; máximo $=2.02)$ y el ancho promedio de la fronda fue de $1.13 \mathrm{~mm}$ (mínimo=0.89; máximo=1.52). Por el largo de la fronda, el número de grupos que forman y la proporción de la distancia que ocupa la nervadura entre el nudo y el ápice, la planta colectada es distinta a $L$. valdiviana (especie que tiene un largo entre 2 y 5 $\mathrm{mm}$, forma grupos entre 4 y 8 frondas y tiene una nervadura que sobrepasa los tres cuartos de la distancia entre el nudo y el ápice de la fronda; León 1993, Armstrong 2013).

Material estudiado: Lima: Huaura: Lomas de Lachay, 385 m, 14/11/15. H. Aponte 272 (USM).

\section{Discusión y conclusiones.}

Siendo esta planta propia de ecosistemas acuáticos, su presencia en esta loma ha sido probablemente facilitada por algún animal que la ha transportado hasta esta localidad. Cerca de las Lomas de Lachay se encuentran humedales como la Laguna El Paraíso (Huacho) y el Humedal de Santa Rosa (Chancay) (Figura 1). De estas dos localidades, solo los humedales de Santa Rosa (que se encuentra a $27 \mathrm{~km}$ de distancia) tienen en su lista de especies a $L$. minuta (Aponte \& Cano, 2013). Siendo que algunas especies de aves transportan plantas acuáticas como L. minuta por ornitocoria (Green et al., 2008), es probable que $L$. minuta haya llegado a las lomas de Lachay por medio de alguna especie que circula entre el humedal de Santa Rosa y las Lomas de Lachay (como Numenius phaeopus L., la cual está reportada para Lachay como especie rara, pero que es una especie migratoria frecuente en los humedales costeros como Santa Rosa (INRENA 2002, Tello \& Engblom, 2010).

Este hallazgo sugiere una conexión entre las lomas y los humedales por medio de la visita de aves que comparten ambos ecosistemas. El presente reporte muestra también el gran potencial adaptativo que tiene L. minuta para poder colonizar ecosistemas siempre que se cubran los requerimientos mínimos para su existencia.

\section{Agradecimientos.}

Agradezco a Biólogo Miguel Angel Antonio Astocaza (Jefe de la Reserva Nacional de Lachay) y al guarda parque Catalino Castillo por las facilidades brindadas para la colecta e identificación de la presente especie. A los profesores Wilfredo Ramírez y Gustavo Lértora, así como a los revisores del presente manuscrito por sus valiosos comentarios. 


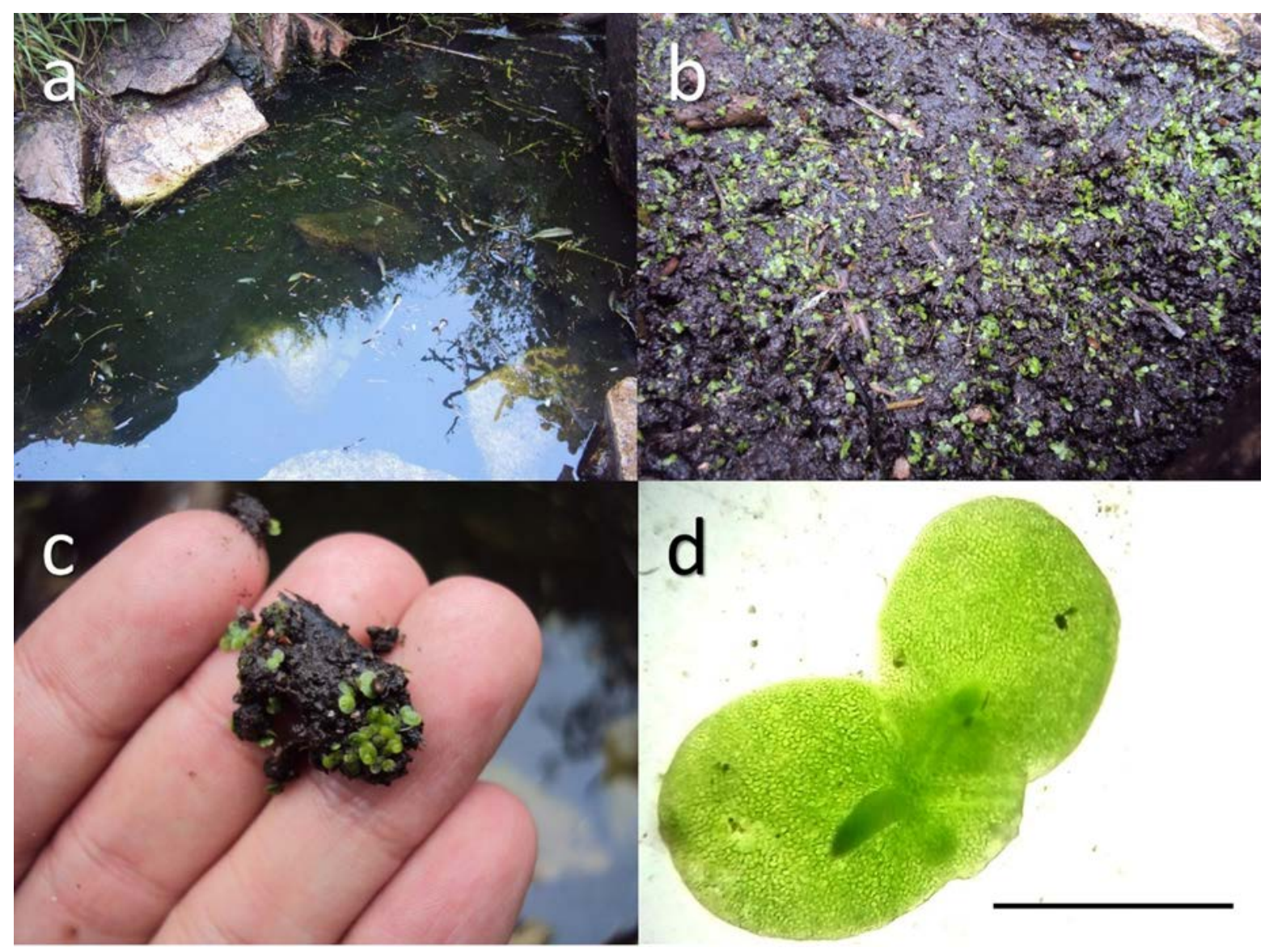

Figura 2. Lemna minuta en las Lomas de Lachay. a) Detalle de El Puquio, fuente de agua donde se encontró la especie mencionada; b) Lemna minuta creciendo en suelo húmedo; c) detalle del suelo con Lemna minuta; d) Toma microscópica de la especie, escala $1 \mathrm{~mm}$.

\section{Literatura citada.}

APG III, The Angiosperm Phylogeny Group. 2009. An update of the Angiosperm Phylogeny Group classification for the orders and families of flowering plants: APG III. Journal of the Linnean Society, 161, 105-21.

Aponte H. \& Cano A. 2013. Estudio Florístico Comparativo de Seis Humedales de la Costa deLima (Perú): Actualización y Nuevos Retos para su Conservación. Revista Latinoamericana de Conservación, 3, 15-27.

Aponte H., Jiménez R. \& Alcántara B. 2012. Challenges for management and conservation of Santa Rosa Wetland (Lima - Peru). Cientifica, 9(3), 257-264.

Armstrong W.P. 2013. On-Line Key to the Genus Lemna. Lemnaceae. Disponible en: http://waynesword.palomar.edu/1lekey.htm. Revisado el 20/4/2016

Brako L. \& J. Zarucchi. 1993. Catálogo de las Angiospermas y Gimnospermas del Perú. Vol. 45. Missouri: Missouri Botanical Garden.

Bridson D.M. \& Forman L. 1998. The Herbarium Handbook, 3rd ed. Royal Botanic Gardens, Kew.

Cano A.C., Roque J., Arakaki M., Arana C., Torre M.L., Llerena N. \& Refulio, N. 1999. Diversidad floristica de las lomas de Lachay (Lima) durante el evento 'El Niño 1997-98’. Revista Peruana de Biología, 6, 125 - 132.
Dillon M.O., Leiva González S., Zapata Cruz M., Lezama Asencio P. \& Quipuscoa Silvestre, V. 2011. Floristic checklist of the Peruvian Lomas Formations. Arnaldoa, 18, 7-32.

Green, A. J., K. M. Jenkins, D. Bell, P. J. Morris, \& R. T. Kingsford. 2008. The Potential Role of Waterbirds in Dispersing Invertebrates and Plants in Arid Australia. Freshwater Biology, 53 (2), 380-92.

Instituto Nacional de Recursos Naturales (INRENA). 2002. Plan Maestro de la Reserva Nacional de Lachay. INRENA. $61 \mathrm{pp}$.

León B. 1993. Catálogo anotado de las fanerógamas acuáticas del Perú. Las Plantas Vasculares en las Aguas Continentales del Perú Travaux de l'Institut Francais d'Etudes Andines. (eds F. Kahn), B. León), \& K. Young), pp. 11-128. IFEA (Institut Francais d'Etudes Andines), Lima-Peru.

Tello A. \& G. Engblom. 2010. Lista de especies de los humedales de la Región Lima: Aves. En Humedales de la Región Lima, Guía de su fauna y flora silvestres, editado por Alejandro Tello y Luis Castillo, 87-90. Lima - Perú: Gobierno Regional de Lima.

Vilà M. \& J. Weiner. 2004. Are Invasive Plant Species Better Competitors than Native Plant Species? Evidence from Pair-Wise Experiments. Oikos 105 (2), 229-38. 
${ }^{1}$ Facultad de Ciencias Biológicas y Veterinarias. Escuela de Biología Marina. Universidad Científica del Sur. Av. Antigua Carretera Panamericana Sur km 19 Villa El Salvador. - Lima 42, Perú. haponteu@yahoo.fr

${ }^{2}$ Museo de Historia Natural, Universidad Nacional Mayor de San Marcos. Avenida Arenales 1256, Jesús María Lima. Apartado 14-0434, Lima 14, Perú. 